\title{
Knowledge and Attitudes of Nurses towards Health Care Associated Infections in Lahore, Pakistan
}

\author{
Article by ${ }^{1}$ Muhammad Afzal ${ }^{*},{ }^{2}$ Ali Waqas, ${ }^{3}$ Muhammad Sabir, ${ }^{4}$ Shazma Ahmad \\ Khan, ${ }^{5}$ Aminullah \\ ${ }^{1}$ Assistant Professor, The University of Lahore, Pakistan \\ ${ }^{2}$ Visiting Lecturer, The University of Lahore, Pakistan \\ ${ }^{3}$ Lecturer, The University of Lahore, Pakistan \\ ${ }^{4}$ Visiting Lecturer, The University of Lahore, Pakistan \\ ${ }^{5}$ BSN student, The University of Lahore, Pakistan \\ Email: muhammad.afzal@lsn.uol.edu.pk
}

\begin{abstract}
This study assesses the knowledge \& attitudes of nurses regarding standard precautions about health care associated infections (HAIs) among the different hospitals of Lahore, Pakistan. Exposure to the body fluids and blood is the main sources for HAIs to the patients. Health care associated infection is a critical issue in the health care facilities because it causes mortality and infection among the hospitalized patients and health care workers. The World Health organization reported that ninety percent of infections among health care workers (HCWs) are associated with the occupational exposure to the body fluids and blood in the developing countries (Wilburn, et al., 2013). Standard precautions are used to provide the protection to health care providers from damages and to stop spread of nosocomial infection. Standard precautions recommend the proper hand hygiene before and after the procedure while contact with the patients (Boyce, et al., 2013).

The current study is of quantitative and cross-sectional design. Target population of the current study was 600 nursing staff of two hospitals of Lahore, Pakistan in which one is public hospital and other one is private hospital. The sample size of the current study was 172 which were calculated by using Slovin's formula. SPSS 20 was used for the data analysis.

The current study result shows that nurses have adequate knowledge regarding standard precautions of HAIs but lack the intention about precautions of HAIs. Thus, it is concluded that low level attitude of nursing staff regarding standards precautions about HAIs was assessed which cause infections to the health care providers and patients.
\end{abstract}

Keywords: Knowledge, Attitudes, Nurses, Standard precautions and Healthcare associated infections (HAIs).

\section{Introduction}

Healthy lifestyle is the life long effort to balance the activities of our life at every movement. Therefore, many situations may affect this balance and deprive our life from happiness. Among these situations, exposure to the body fluids and blood are the main sources for healthcare associated infections (HAIs). HAIs is a critical issue in the health care facilities because of its common cause of mortality and infection among the hospitalized patients and health care providers. Healthcare facilities are classified into biological, chemical, ergonomic, mechanical, physical and psychosocial.

The World Health Organization reported that ninety percent of infections among health care workers (HCWs) are attributed to the occupational exposure to the body fluids and blood in the developing countries (Wilburn, et al., 2013).

Healthcare associated infections (HAIs) can be defined as a systemic or localized condition that happens from an adverse exposure or reaction of the presence of an infectious agent that happens during hospital admission and there is no evidence that the infection was incubating 
South American Journal of Nursing

Volume 2, Issue 1, 2016

or present at the time of admission. Eriksen (2012) stated that incidence of health care associated infections are increased in Europe and United States within last 5 years.

Mbaisi (2013) investigated the health care sector of Kenya and emphasizes that the accidental occupational contact of healthcare workers to blood and body fluids subsequent to skin injury or mucous membrane contact comprises a risk for transmission of blood-borne pathogens. Moreover, the study describes that $25 \%$ of health care workers reported that they have been exposed to the blood and body fluids, overall 55.5\% nurses were commonly injured during injection procedure to the patients, $78.9 \%$ during stitching, $25 \%$ laboratory personnel during blood specimen collection while $57.1 \%$ supportive staff during cleaning.

Afridi (2013) reported the reasons for needle stick injuries (NSIs) in Pakistan that $42 \%$ of nurses exposed to the needle stick injury during injecting medicine and drawing blood and $37 \%$ of nurses exposed to needle stick injury during two-handed recapping of needle. Furthermore, 34\% of the nurses were vaccinated against hepatitis B infection. However, nurses had inadequate facilities at workplace concerning standard precautions such as $40 \%$ of nurses have accessibility of gloves / protective cloths and $10 \%$ of nurses fulfil protocols / infection control guidelines.

Serrano (2016) noted that overall prevalence rate of healthcare associated infections (HAIs) was $10.2 \%$. Palliative care units and subacute care units have highest rate of HAIs that is $22.3 \%$ and $18.7 \%$ respectively. However, common infections were respiratory tract infection and urinary tract infection in long term care facilities.

Siegel (2012) emphasizes that proper implementation of standard precaution decreases the rate of healthcare associated infections (HAIs) from both unknown sources and expected sources in the healthcare setting. Moreover, implementation of standard precautions decreases the transmission of respiratory infections, human Immunodeficiency virus (HIV), hepatitis B (HBV) and other pathogens which grow in the blood and body fluids and minimize the risk of HAIs among nurses and other health care providers.

Magill (2014) highlighted that health care associated infections (HAIs) are increasing in U.S as $4.0 \%$ of the patients keep at least 1 health care-associated infection. Pneumonia and surgical-site infection were most common, subsequently gastrointestinal infection, primary bloodstream infection and urinary tract infection.

\section{Study purpose}

The purpose of this study is to assess the knowledge \& attitude of nurse regarding standard precautions of Healthcare Associated Infections (HAIs) in different health care facilities of Lahore, Pakistan.

\section{Significance of the study}

This study provides awareness among the health care providers in different hospitals \& explores the knowledge and attitudes of nurses' related standard precautions towards healthcare associated infections and its implementations. The study results will be helpful to the policy makers of different hospitals to develop the strategies for enhancement of knowledge and attitude of nurses. Similarly, the study will contribute to reduce the HAIs.

\section{Objectives of the study}

1) To determine the level of knowledge of nurses regarding standards precautions about healthcare associated infections (HAIs) in hospitals of Lahore, Pakistan.

2) To determine the level of attitude of nurses regarding standards precautions about healthcare associated infections (HAIs) in hospitals of Lahore, Pakistan.

\section{Literature review}

Hand hygiene is acknowledged as one of the most important strategies for preventing healthcare associated infections (HAIs). During observations, it has been noticed that $40 \%$ of health care workers (HCW) adhere with contact precautions or use of gloves, masks and 
gowns while patients care (Septimus, et al., 2014). Furthermore, implementation of universal precautions has decreased the HAIs such as $44 \%$ reduction in all-cause bloodstream infection and 37\% of MRSA clinical culture rates (Septimus, et al., 2014).

In addition, environmental contamination is the major determinant of transmission of HAIs to healthcare workers and patients. Morgan (2012) found that during routine clinical care of patients, $62 \%$ of HCWs who entered the patients' room wearing the contaminated gloves, mask, and gown. In developing countries, environmental contamination, malnutrition and frequently usage of invasive devices are major cause of HAIs. It has observed that invasive procedure or devices cause 73\% HAIs in the hospitalized patients (Polin, et al., 2012). Moreover, proper utilization of universal precautions in ICUs decreases $40 \%$ to $50 \%$ of HAIs (Polin, et al., 2012).

The prevalence rate for HAIs has increased globally over the past 2 decades due to invasive procedures or devices such as mechanical ventilator, central venous catheter (CVC), arterial catheter, indwelling urinary catheter and wound drainage tube. Invasive procedure causing overall $55 \%$ of HAIs in which urinary tract infection is $54.8 \%$, blood stream infection is $30.6 \%$, surgical site infection is $6.6 \%$, pneumonia is $4.5 \%$ and other site infection is $3.5 \%$ (Yang, et al., 2013).

Improvement in the hand hygiene of HCWs decreases the transmission of pathogens, therefore, hand hygiene is widely accepted as a foundation to avoid the infections. Ellingson (2014) mentioned that adherence with 5 practices of hand hygiene reduces more than $50 \%$ of transmission of pathogens to the ICU patients. These practices are: wash hands before (1) touching a patient, (2) clean / aseptic procedure, and after (3) body fluid exposure, (4) touching a patient and (5) touching patient surrounding.

A number of strategies have been investigated for the prevention of HAIs spread among nurses and hospitalized patients in which hand washing has been proven to be a significant component of interventions for the reduction of HAIs. Angelis (2014) analyzed that proper utilization of hand hygiene measures significantly (47\%) decreases the rate of HAIs among nurses and patients.

Riven (2015) assessed the range of microorganisms found on noninvasive portable clinical items potentially shared among patients (NPIs) and evaluate the evidence regarding the potential for cross-transmission of microorganisms between NPIs and hospitalized patients in non-outbreak conditions. Subsequently, the study result shows that those rates of NPI contamination ranged from $23 \%$ to $90 \%$. Normal skin or environmental floras were found on almost all positive cultures.

Kirk (2016) emphasizes that $25 \%$ of health care providers follow the standard precautions during patient care. However, as compared to knowledge the attitude of nurses was disappointingly less regarding the precautions about the HAIs.

\section{Research methodology}

Cross sectional descriptive study design was used to assess the knowledge and attitude of nurses regarding standards precaution of healthcare associated infection in the hospitals of Lahore. The data were obtained through the adapted questionnaire of Knowledge and attitude regarding HAIs of Parmeggiani (2010) which is based on 5 point Likert scale. Target population of the study was 600 registered nurses of the hospitals of Lahore, Pakistan. The data was collected from two hospitals (Nawaz Sharif Social Security Hospital \& Doctors Hospital) of Lahore, Pakistan. Sample size of the current study was calculated by using Slovin's formula ( $\mathrm{n}=172)$, so, self-administered questionnaire was distributed to 172 nurses by simple random sampling technique. The inclusion criteria for this study were registered nurses while the student nurses and head nurses excluded. Period of the study was February 2016 to May 2016. 
South American Journal of Nursing

Volume 2, Issue 1, 2016

\section{Data analysis \& results}

Reliability of the tool checked through Cronbach's alpha. Data was collected from both male and female nurses in which $83.7 \%(\mathrm{n}=144)$ were females and $16.3 \%(\mathrm{n}=28)$ were males. Majority of nurses that is $79.6 \%(\mathrm{n}=132)$ of participants belong to age group $20-35$ years in which $59.3 \%$ were single and only $40.7 \%$ were married. Data shows that $89.5 \%(n=154)$ nurses have General Nursing Diploma and 10.5\% ( $n=18)$ has BS Nursing degree.

Table 1. Wearing gloves, mask, and protective eyewear are a HAIs control measures

\begin{tabular}{|ll|l|l|l|l|}
\hline & Frequency & Percent & $\begin{array}{l}\text { Valid } \\
\text { Percent }\end{array}$ & $\begin{array}{l}\text { Cumulative } \\
\text { Percent }\end{array}$ \\
\hline \multirow{6}{*}{ Valid } & Strongly Disagree & 5 & 2.9 & 2.9 & 2.9 \\
& Disagree & 15 & 8.7 & 8.7 & 11.6 \\
Neutral & 44 & 25.6 & 25.6 & 37.2 \\
& Agree & 51 & 29.7 & 29.7 & 66.9 \\
Strongly Agree & 57 & 33.1 & 33.1 & 100.0 \\
Total & 172 & 100.0 & 100.0 & \\
\hline
\end{tabular}

Responses regarding knowledge of Health Care Workers (HCWs) or nurses are reported in Table1. Majority 62.8\% $(\mathrm{n}=108)$ of nurses were aware that wearing of gloves, mask, and protective eyewear reduce the risk of HAIs although $11.6 \%(n=20)$ did not know that wearing of gloves, mask, and protective eyewear control the HAIs.

Table 2. Invasive procedures are a risk factor for HAIs

\begin{tabular}{|ll|l|l|l|l|}
\hline & Frequency & Percent & Valid Percent & $\begin{array}{l}\text { Cumulative } \\
\text { Percent }\end{array}$ \\
\hline \multirow{4}{*}{ Valid } & Strongly Disagree & 8 & 4.7 & 4.7 & 4.7 \\
& Disagree & 11 & 6.4 & 6.4 & 11.1 \\
Neutral & 47 & 27.3 & 27.3 & 38.4 \\
& Agree & 46 & 26.7 & 26.7 & 65.1 \\
& Strongly Agree & 60 & 34.9 & 34.9 & 100.0 \\
& Total & 172 & 100.0 & 100.0 & \\
\hline
\end{tabular}

In Table 2, 61.6\% $(\mathrm{n}=106)$ of the respondents knew that invasive procedures are a risk factor for HAIs while $11.1 \%(\mathrm{n}=19)$ disagree that invasive procedures are risk factors for HAIs.

Table 3. HCWs' hands are a vehicle for transmission of nosocomial pathogens

\begin{tabular}{|l|l|l|l|l|}
\hline & Frequency & Percent & Valid Percent & $\begin{array}{l}\text { Cumulative } \\
\text { Percent }\end{array}$ \\
\hline \multirow{2}{*}{ Valid } & 7 & 4.1 & 4.1 & 4.1 \\
Disagree & 17 & 9.9 & 9.9 & 14.0 \\
Neutral & 45 & 26.2 & 26.2 & 40.1 \\
Agree & 54 & 31.4 & 31.4 & 71.5 \\
Strongly Agree & 49 & 28.5 & 28.5 & 100.0 \\
Total & 172 & 100.0 & 100.0 & \\
\hline
\end{tabular}

Table 3 depicts that preponderance 59.9\% $(n=103)$ believed that HCWs' hands are a vehicle for transmission of nosocomial pathogens although $14 \%(n=24)$ respondents believed that HCWs are not accountable in transmission of nosocomial infection to the patients. 
Table 4. Hands hygiene measures reduce the risk of HAIs among patients

\begin{tabular}{|cl|l|l|l|l|}
\hline & Frequency & Percent & Valid Percent & $\begin{array}{l}\text { Cumulative } \\
\text { Percent }\end{array}$ \\
\hline \multirow{2}{*}{ Valid } & Strongly Disagree & 10 & 5.8 & 5.8 & 5.8 \\
Disagree & 20 & 11.6 & 11.6 & 17.4 \\
Neutral & 41 & 23.8 & 23.8 & 41.3 \\
Agree & 52 & 30.2 & 30.2 & 71.5 \\
Strongly Agree & 49 & 28.5 & 28.5 & 100.0 \\
Total & 172 & 100.0 & 100.0 & \\
\hline
\end{tabular}

Concerning the perceived risk of acquiring healthcare associated infections (HAIs), health care workers (HCWs) and nurses are at high risk for transfer of infections to the patients and among other health care workers. Table 4 shows that $57.8 \%(n=101)$ of nurses believes that good hand hygiene of health care workers (HCWs) reduce the risk of HAIs among patients while $17.4 \%(n=30)$ did not know that hand hygiene of health care workers (HCWs) reduce the risk of HAIs.

Table 5. Hands hygiene measures reduce the risk of HAIs among HCWs

\begin{tabular}{|ll|l|l|l|l|}
\hline & Frequency & Percent & Valid Percent & $\begin{array}{l}\text { Cumulative } \\
\text { Percent }\end{array}$ \\
\hline \multirow{6}{*}{ Valid } & Strongly Disagree & 11 & 6.4 & 6.4 & 6.4 \\
& Disagree & 17 & 9.9 & 9.9 & 16.3 \\
Neutral & 41 & 23.8 & 23.8 & 40.1 \\
& Agree & 56 & 32.6 & 32.6 & 72.7 \\
Strongly Agree & 47 & 27.3 & 27.3 & 100.0 \\
Total & 172 & 100.0 & 100.0 & \\
\hline
\end{tabular}

Table 5 shows that nurses had an extremely positive attitude, so, 59.9\% $(n=103)$ of total emphasize that guideline of HAIs should be followed because hands hygiene measures reduce the risk of HAIs among HCWs, however 16.3\% (n=28) nurses did not believe it.

Table 6. Aprons and face masks should be worn in procedures where splash/spill of blood is likely

\begin{tabular}{|ll|l|l|l|l|}
\hline & Frequency & Percent & Valid Percent & $\begin{array}{l}\text { Cumulative } \\
\text { Percent }\end{array}$ \\
\hline \multirow{4}{*}{ Valid } & Strongly Disagree & 9 & 5.2 & 5.2 & 5.2 \\
& Disagree & 13 & 7.6 & 7.6 & 12.8 \\
Neutral & 41 & 23.8 & 23.8 & 36.6 \\
& Agree & 67 & 39.0 & 39.0 & 75.6 \\
& Strongly Agree & 42 & 24.4 & 24.4 & 100.0 \\
& Total & 172 & 100.0 & 100.0 & \\
\hline
\end{tabular}

Table 6 shows that $63.4 \%(n=109)$ of nurses perceived that wearing of aprons and face masks decrease the HAIs during procedures where splash/spill of blood is likely, though $12.8 \%(n=22)$ of nurses do not distinguish that proper wearing of aprons and face masks decrease the HAIs during procedures where splash/spill of blood is likely.

\section{Discussion}

The current study examines the level of knowledge \& attitudes of nurses of Lahore, Pakistan about healthcare associated infections (HAIs). The result proves that nurses and HCWs have enough knowledge regarding hand hygiene and wearing of gloves, masks, and 
South American Journal of Nursing

Volume 2, Issue 1, 2016

protective eyewear during patient care as tools to control the HAIs. Similarly, the study results of Asadollahi (2015) and Hosseinialhashemi (2015) that nurses have sufficient knowledge about hand hygiene and wearing of protective measures but they need to more emphasize on the training of hand hygiene for the improvement in the attitudes and practice. Similarly, Kirk (2016) emphasizes that nurses and doctors have more knowledge as compare to the attitude regarding prevention of HAIs.

Numerous studies support that the effect of knowledge and attitude of nurses and health care workers to reduce HAIs transmitted by hand. HAIs is critical to the healthcare facilities and increases the risks, prevalence of diseases and hospital costs. HAIs results in 4 to 5 hospitalization days and adds significant cost to the hospitals. However, hand hygiene is the most valuable means of prevention of HAIs. The current study results proved that nurses and HCWs have knowledge regarding importance of hand hygiene in HAIs but only $60 \%$ health care workers focusing on hand hygiene practices, therefore, HAIs are high in developing countries. Similarly, Jain (2015) analyzed the knowledge and attitude of doctors and nurses towards HAIs and found that only $57 \%$ of the respondents fulfil hand hygiene measures during patient care and invasive procedures, therefore, HAIs also high in such hospitals. The results of this study show that $57.6 \%$ of nurses agree that healthcare associated infections are control by proper measure, $6 \%$ participants were disagreeing and rest of the participants have given neutral response.

The healthcare providers in any healthcare systems should be well aware of effectiveness of HAIs control measures. The poor attitude of HCWs and nurses indicates a strong need of on job healthcare providers (nurses) trainings in the developing countries to control HAIs (Kanwar, et al., 2015).

\section{Conclusion}

Standard precautions are the repetitive action that may lead to the foundation of a habit and it may influence behavior which depends on the knowledge. Nurses and health care workers (HCWs) show high level of knowledge regarding prevention of healthcare associated infections (HAIs), but low attitude regarding standard precautions about HAIs. It is urgency to start on job training programs for HCWs for bringing change in their attitude. Furthermore, to motivate HCWs through different incentives for implementation of standard precautions because this practice ultimately decreases the rate of HAIs and patient stay in the hospital.

\section{Limitations}

There are some limitations of the current study. First, it offers as a cross-sectional study, only depends upon specific questions. Target population was nurses and low health care workers; therefore it ignores the participation of doctors and pharmacist due to shortage of time. Second limitation is the potential recording bias associated with the self-administered questionnaire. Third, the current study only investigates two hospitals of Lahore due to the time constraints which may not be generalizable to the other parts of the country.

\section{Recommendation}

The findings of the current study provide the valuable baseline data for further investigation and intervention. Future researchers may conduct interventional and qualitative study on large scale and also include the physicians, surgeons, pharmacist and other health care workers. Authorities should enhance the knowledge and attitude of health care team for the attainment of better result. The future research may do the comparison of level of knowledge and attitude among the nurses of public and private universities. 


\section{References}

[1.] Asadollahi, M., Bostanabad, M. A., Jebraili, M., Mahallei, M., Rasooli, A. S., \& Abdolalipour, M. (2015). Nurses' knowledge regarding hand hygiene and its individual and organizational predictors. Journal of caring sciences, 4(1), 45.

[2.] Boyce, J. M., \& Pittet, D. (2002). Guideline for hand hygiene in health-care settings: recommendations of the Healthcare Infection Control Practices Advisory Committee and the HICPAC/SHEA/APIC/IDSA Hand Hygiene Task Force. American journal of infection control, 30(8), S1-S46.

[3.] De Angelis, G., Cataldo, M. A., De Waure, C., Venturiello, S., La Torre, G., Cauda, R., \& Tacconelli, E. (2014). Infection control and prevention measures to reduce the spread of vancomycinresistant enterococci in hospitalized patients: a systematic review and meta-analysis. Journal of Antimicrobial Chemotherapy, dkt525.

[4.] Ellingson, K., Haas, J. P., Aiello, A. E., Kusek, L., Maragakis, L. L., Olmsted, R. N., \& VanAmringe, M. (2014). Strategies to prevent healthcare-associated infections through hand hygiene. Infection Control \& Hospital Epidemiology, 35(08), 937-960.

[5.] Eriksen, H. M., Iversen, B. G., \& Aavitsland, P. (2005). Prevalence of nosocomial infections in hospitals in Norway, 2002 and 2003. Journal of Hospital Infection, 60(1), 40-45.

[6.] Europe, W. (2012). Management of Hepatitis C and HIV co-infection-Clinical protocol for the WHO European Region. Last accessed September, 12.

[7.] Hosseinialhashemi, M., Kermani, F. S., Palenik, C. J., Pourasghari, H., \& Askarian, M. (2015). Knowledge, attitudes, and practices of health care personnel concerning hand hygiene in Shiraz University of Medical Sciences hospitals, 2013-2014. American journal of infection control, 43(9), 1009-1011.

[8.] Jain, M., Dogra, V., Mishra, B., Thakur, A., \& Loomba, P. S. (2015). Knowledge and attitude of doctors and nurses regarding indication for catheterization and prevention of catheter-associated urinary tract infection in a tertiary care hospital. Indian journal of critical care medicine: peer-reviewed, official publication of Indian Society of Critical Care Medicine, 19(2), 76.

[9.] Kanwar, V., Sood, A., Gupta, P. K., \& Salaria, N. (2015). Knowledge regarding infection control practices among nurses in rural public health settings: an emerging public health concern in India. Int $\mathrm{J}$ Health Sci Res, 5(2), 282-287.

[10.] Kirk, J., Kendall, A., Marx, J. F., Pincock, T., Young, E., Hughes, J. M., \& Landers, T. (2016). Point of care hand hygiene-where's the rub? A survey of US and Canadian health care workers' knowledge, attitudes, and practices. American journal of infection control.

[11.] Magill, S. S., Edwards, J. R., Bamberg, W., Beldavs, Z. G., Dumyati, G., Kainer, M. A., \& Ray, S. M. (2014). Multistate point-prevalence survey of health care-associated infections. New England Journal of Medicine, 370(13), 1198-1208.

[12.] Mbaisi, E. M., Wanzala, P., \& Omolo, J. (2013). Prevalence and factors associated with percutaneous injuries and splash exposures among health-care workers in a provincial hospital, Kenya, 2010. Pan African Medical Journal, 14(1).

[13.] Mody, L., Meddings, J., Edson, B. S., McNamara, S. E., Trautner, B. W., Stone, N. D., \& Saint, S. (2015). Enhancing resident safety by preventing healthcare-associated infection: a national initiative to reduce catheter-associated urinary tract infections in nursing homes. Clinical Infectious Diseases, 61(1), 86-94.

[14.] Morgan, D. J., Rogawski, E., Thom, K. A., Johnson, J. K., Perencevich, E. N., Shardell, M., \& Harris, A. D. (2012). Transfer of multidrug-resistant bacteria to healthcare workers' gloves and gowns after patient contact increases with environmental contamination. Critical care medicine, 40(4), 1045.

[15.] Parmeggiani, C., Abbate, R., Marinelli, P., \& Angelillo, I. F. (2010). Healthcare workers and health care-associated infections: knowledge, attitudes, and behavior in emergency departments in Italy. BMC infectious diseases, 10(1), 1.

[16.] Polin, R. A., Denson, S., Brady, M. T., Papile, L. A., Baley, J. E., Carlo, W. A., \& Byington, C. L. (2012). Strategies for prevention of health care-associated infections in the NICU. Pediatrics, 129(4), e1085-e1093. 
South American Journal of Nursing

Volume 2, Issue 1, 2016

[17.] Septimus, E., Weinstein, R. A., Perl, T. M., Goldmann, D. A., \& Yokoe, D. S. (2014). Approaches for preventing healthcare-associated infections: go long or go wide?. Infection Control \& Hospital Epidemiology, 35(07), 797-801.

[18.] Serrano, M., Barcenilla, F., Limón, E., Pujol, M., \& Gudiol, F. (2016). [Prevalence of healthcareassociated infections in long-term care facilities in Catalonia. VINCat Program]. Enfermedades infecciosas y microbiologia clinica.

[19.] Siegel, J., et al. (2012). Guideline for isolation precautions: preventing transmission of infectious agents in health care settings: American journal of infectio control.

[20.] Wilburn SQ, Eijkemans G. (2013). Preventing needlestick injuries among healthcare workers: a WHO-ICN collaboration.

[21.] Yang, S. P., Chen, Y. Y., Hsu, H. S., Wang, F. D., Chen, L. Y., \& Fung, C. P. (2013). A risk factor analysis of healthcare-associated fungal infections in an intensive care unit: a retrospective cohort study. BMC infectious diseases, 13(1), 1. 